\title{
Vorzeitiger Blasensprung: Abwarten lohnt sich
}

\author{
Bei einem Blasensprung in der 34. bis 37. Schwangerschaftswoche lautet \\ die Frage: Sofort entbinden oder abwarten? Eine Gruppe von Neonatologen \\ und Gynäkologen glaubt die Antwort zu kennen.
}

B ei einem vorzeitigen Blasensprung bis zur 30. Schwangerschaftswoche (SSW) sollte besser abgewartet werden, sofern sich keine sonstigen Komplikationen einstellen. Ist das Kind bereits voll ausgetragen, spricht die Datenlage für eine umgehende Entbindung, weil sie das Infektionsrisiko für die Mutter senkt und keine Nachteile für ihr Baby zu erwarten sind. Unklar ist die Lage, wenn keiner der beiden Fälle vorliegt. Ist der Fetus noch nicht voll ausgereift, drohen kurz- und langfristig gesundheitliche Nachteile. Abzuwägen ist dies gegen $\mathrm{Ge}$ fahren wie Plazentaablösung, aufsteigende Infektion und Nabelschnurvorfall.

In der Studie „Preterm Prelabour Rupture Of Membranes near Term“ (PPROMT) haben Mediziner aus elf Ländern und 65 Zentren untersucht, welche Strategie - Entbinden oder Abwarten - bei vorzeitigem Blasensprung in der 34.-36. SSW plus sechs Tage die besseren Ergebnisse liefert. Mehr als 1.800 mit Einlingen Schwangere wurden dafür auf zwei Gruppen verteilt: 900 wurden sofort entbunden, bei anderen 900 wartete man bis zur Spontangeburt $\mathrm{ab}$, sofern sich keine andere Indikation ergab.

Die Rate an Neonatalsepsis - primärer Studienendpunkt - unterschied sich in beiden Gruppen nicht signifikant (3\% unter Abwarten, $2 \%$ bei Entbindung). Auch im sekundären Gesamtendpunkt (Sepsis, Beatmungspflicht über mind. 24 Stunden, Totgeburt oder Tod des Neugeborenen) waren die Raten mit $7 \%$ und $8 \%$ ähnlich. Dagegen gab es bedeutende Unterschiede in der Rate von Atemnotsyndromen (5\% unter Abwarten vs. $8 \%$ bei Entbindung) und maschineller Beatmung (9\% vs. $12 \%$ ). Zudem lagen die sofort entbundenen Kinder durchschnittlich länger auf der Intensivstation (2 vs. 4 Tage). Andererseits ereigneten sich unter abwartender Haltung mehr anteoder intrapartale Blutungen (5\% vs. $3 \%$ ) und die Mütter bekamen häufiger Fieber ( $2 \%$ vs. $1 \%)$.
In beiden Gruppen kamen fast alle Kinder vor der 38. Woche zur Welt. Laut Ergebnissen der PPROMT-Studie hat das Abwarten zumindest für das Kind mehr Vor- als Nachteile. Deshalb raten die Autoren entgegen den Empfehlungen in Leitlinien, besser abzuwarten als eine sofortige Entbindung einzuleiten. Allerdings müssten die Frauen hinsichtlich Blutungsrisiko und Fieber gut überwacht werden. Die PPROMT-Ergebnisse stellen auch die derzeit in Überarbeitung befindlichen Empfehlungen der Deutschen Gesellschaft für Gynäkologie und Geburtshilfe infrage. Dort heißt es, bei vorzeitigem Blasensprung nach der 34 . SSW sei die Geburt nach 12-24 Stunden einzuleiten, falls bis dahin keine spontanen Wehen eingesetzt hätten - auch dann, wenn keine Anzeichen für ein Amnioninfektionssyndrom vorlägen.

Dr. Robert Bublak

Morris J M et al Immediate delivery compared with expectant management after preterm prelabour rupture of the membranes close to term (PPROMT trial): a randomised controlled trial. Lancet 2015, online 9. November; doi: 10.1016/ S0140-6736(15)00724-2

\section{Kommentar}

Septische Infektionen des Neu- und Frühgeborenen sind in Abhängigkeit vom erreichten Gestationsalter mit die wichtigsten Faktoren für Langzeitmorbidität und -mortalität von Neugeborenen. Etablierte Management-Strategien beim Hauptrisikofaktor für septische Infektionen von neugeborenen Kindern und frühzeitigem Blasensprung sind tradiert und weniger evidenzbasiert. Risiken der Frühgeburtlichkeit an sich, im Verhältnis zum Risiko septischer Komplikationen beim Neugeborenen, basieren teilweise auf sehr alten, nicht mehr gültigen epidemiologischen Daten.

In der Realität besteht aber die paradoxe Situation, dass beim höchsten Risiko für septische Komplikationen die Letalität von Frühgeborenen der 23.-25. SSW fast zu $80 \%$ infektionsbedingt und nicht mehr allein RDS(,, respiratory distress syndrome")-bedingt ist. Die unter

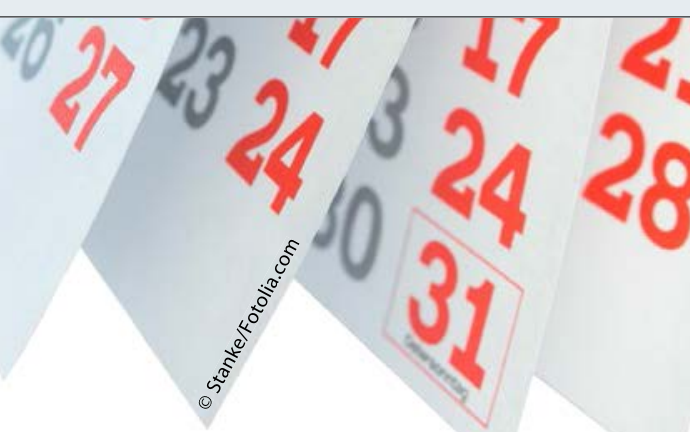

australischer Federführung durchgeführte PPROMT-Studie ist daher außerordentlich verdienstvoll. Hier wurden Entbindungen in elf verschiedenen Ländern mit unterschiedlichem sozioökonomischem Background kontrolliert durchgeführt. Aufwand und Management einer solchen klinischen Studie sind kaum abschätzbar. Schwangerschaften zwischen der 34. und 36. SSW wurden bei vorzeitigem Blasensprung (der allerdings in einigen Fällen schon in der 28. Woche erfolgte) hinsichtlich abwartenden oder sofortigen Vorgehen randomisiert. Es zeigten sich bezüglich des primären Studienendpunktes des sehr gut und präzise definierten Kriteriums der neonatalen Sepsis keine signifikanten Unterschiede. Die Sepsisraten in den Gruppen lagen bei $3 \%$, respektive $2 \%$. Auch ein Compound-Endpunkt der Totgeburt, Komplikationen wie maschinelle Beatmung und Aufenthalt auf der Intensivstation berücksichtigte, zeigte keinen Unterschied zwischen der abwartenden und sofortigen Strategie.

Die Studie ist von außerordentlicher Bedeutung für die Konzeption von Leitlinien, die unbedingt mehr als bisher das Gestationsalter und eine klare Risiko-Nutzen-Abwägung berücksichtigen müssen. Hier wird sich bei später Frühgeburtlichkeit vielleicht ein abwartenderes Verhalten durchsetzen als es in der Extrem-Frühgeborenenmedizin sinnvoll ist. Dort gewinnt man mit Prolongation der Schwangerschaft nach Abschluss der Lungenreife gar nichts oder die neonatale Mortalität steigt, wie einige Studien der letzten Jahre zeigten. Im German Neonatal Network allerdings war auch bei sehr kleinen Frühgeborenen kein Unterschied zwischen abwartendem und unverzüglichem Entbindungsmanagement gefunden worden [Hanke et al. PLoS One April 2015 doi:10.1371/journal.0122564.eCollection 2015].

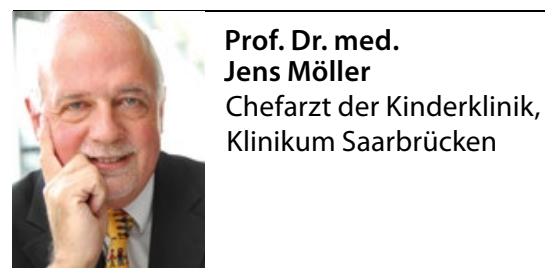

